\title{
Hydraulic Model Calibration and Performance Assessment of Pressure Managed Areas with Multiple Inlets
}

\author{
Attila Bibok ${ }^{1,2^{*}}$, Roland Fülöp \\ 1 Department of Environmental and Sanitary Engineering, Faculty of Civil Engineering, Budapest University of Technology and \\ Economics, Múegyetem rkp. 3, H-1111 Budapest, Hungary \\ 2 Budapest Waterworks, Váci út 182, H-1138 Budapest, Hungary \\ *Corresponding author, e-mail: bibok.attila@epito.bme.hu
}

Received: 23 January 2020, Accepted: 30 March 2020, Published online: 28 April 2020

\begin{abstract}
Pressure management is a widely adopted technique in the toolset of drinking water distribution system operators. It has multiple benefits, like reducing physical losses in pipe networks with excessive leakage, prolong the expected lifetime of the pipes and protecting home appliances from unacceptably high pressure. In some cases, even legislation compliance can be the motivation behind pressure management: It is mandatory to supply water at the customer's connection between 1.5 and 6.0 bar in Hungary since 2011. Diaphragm pressure reducing valves are widespread in the drinking water distribution networks. Although, their sensitivity for gas pocket accumulation in the valve house makes hydraulic calibration of these pressure managed areas a challenging task for hydraulic modelers and network operators. This is especially true when more than one inlet is used to supply the same area in order to increase resilience and flow capacity.

This paper investigates the hydraulic properties of pressure reduced areas with multiple inlet points. Model calibration using a single valve and minor loss was found insufficient because the additional pressure loss referenced to the pressure setting has a nonquadratic relationship with flow-rate on the discharge side under real-life circumstances. This phenomenon can be handled by using a PRV (pressure reducing valve) + GPV (general purpose valve) in series.
\end{abstract}

Keywords

calibration, hydraulic modeling, pressure management, EPANET

\section{Introduction}

The high level of Non-revenue Water is a growing concern for most of the water utility operators. Physical losses are a major component of non-revenue water (up to $40 \%$ ), which can be reduced by pressure reduction [1]. There are various approaches, which were applied in practice [2]. It includes fixed outlet pressure, time-modulated, flow-modulated or critical point pressure modulated methods [3, 4]. The pressure reduction is usually performed by a diaphragm valve or a plunger valve. Diaphragm valves are own-medium controlled, which reduces the necessary infrastructure and costs compared to a plunger valve, which is typically electrically operated [5]. The applied method depends on the characteristics of the pressure managed area including the differences in elevation, the capacity of the water distribution network, available infrastructure at the site, cost of installation and maintenance, and the daily or seasonal changes in the water demand.
The results of pressure management depend on many factors, but $37 \%$ to $83 \%$ minimum night flow reduction can be achieved, based on experiences from practice [6, 7].

The consumer and industrial water prices are fixed and reduced by $20 \%$ by the Hungarian authorities since 2012 . This financial environment increases the demand for higher operational efficiency for utility operators in order to reduce leakages and save on the cost of operation.

There are 48 pressure reduced areas in the drinking water distribution system of Budapest Waterworks, which are supplied by 101 pressure reducing valves. All of the installed pressure reducing valves are diaphragm valves. Most of the pressure reduced areas have at least two inlets to keep the resilience of the system high and to provide continuous water supply even during maintenance and repair tasks. 
Even with these measures, some zones had an increased number of consumer complaints about low service pressure. A well-metered pressure reduced area was investigated in order to find the cause of the increase in complaints.

There are six unknown variables in the aforementioned network layout. Three pressure setting values and three minor headloss coefficients, which assumes a quadratic relationship between head loss and flow-rate. The calibration runs using this conventional setup showed a trade-off between the downstream pressures [8].

This paper investigates a different approach to accurately model the steady-state hydraulics of a multi-inlet pressure reduced zone shown in Fig. 1. The paper also details the operational evaluation and performance assessment of these mechanically operated pressure reducing valves.

\section{Model setup}

A new model setup was proposed in which the minor loss coefficient was substituted by a GPV (general purpose valve). The advantage of this layout is that custom, non-quadratic head loss-flow-rate relationships can be introduced into the model. It is important to note that the GPV is not representing a real valve but is used to introduce the PRV's custom head loss-flow relationship into the model.

This approach increases the dimensionality of the problem by the additional unknown variables. The more detailed the GPV curves, the more complex the model gets as the number of unknown variables increases.

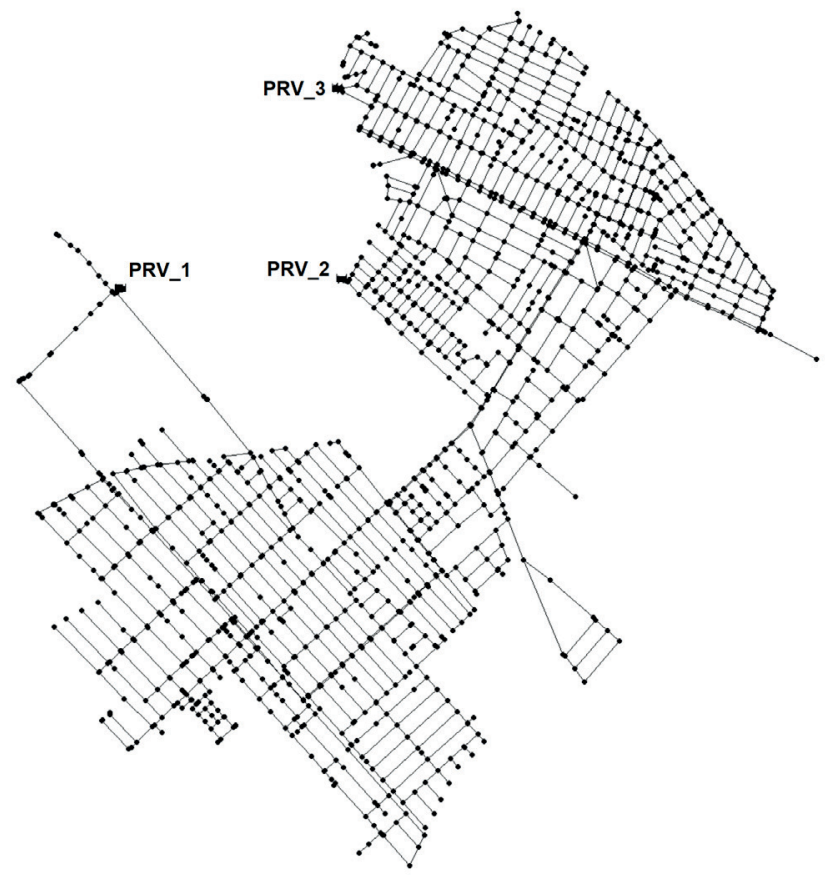

Fig. 1 Network layout of the hydraulic model - Zone 23/1

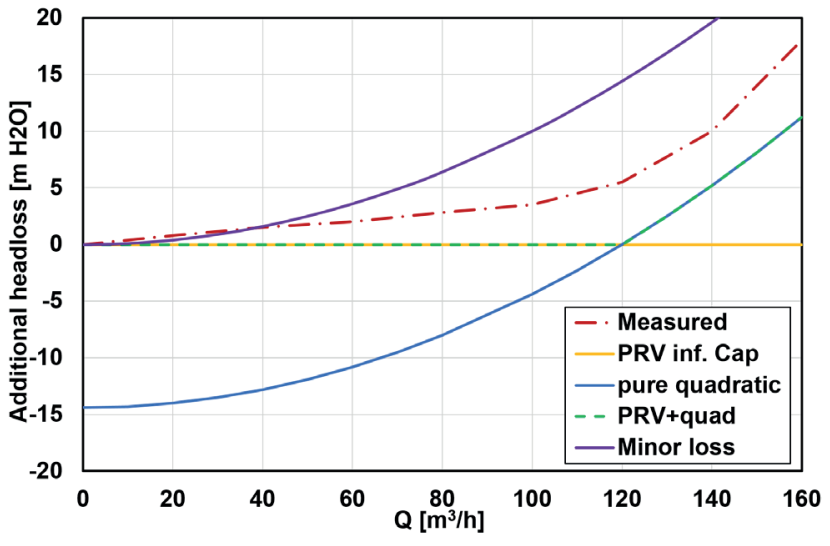

Fig. 2 Different types of PRV head loss curves in hydraulic modeling. 0 is the reference pressure head at valve closure

As Fig. 2 shows, using quadratic loss functions, also known as minor losses, is not suitable to model the hydraulic behavior of a diaphragm pressure reducing valve.

\subsection{PRVs using EPANET 2.0}

The proposed model setup is not specific to a proprietary modeling software or framework. All the widespread commercial and open-source distribution network modeling tools are capable of implementing the proposed PRV scheme. The main reason behind it is the EPANET compatibility because most of the commercial software was forked from EPANET at some point. Fig. 3 also shows the difference between the original modeling approach (above) and the proposed setup (below).

\subsection{PRVs using Bentley's WaterGEMS}

Bentley Systems' WaterGEMS [9] is a commercial, closedsource hydraulic modeling software, which is highly popular among drinking water hydraulics professionals. It is compatible with EPANET input files, but it handles nodes and links slightly differently on a topological level. Valves are considered as nodal objects in WaterGEMS, in contrary to EPANET in which they are links (Fig. 4).

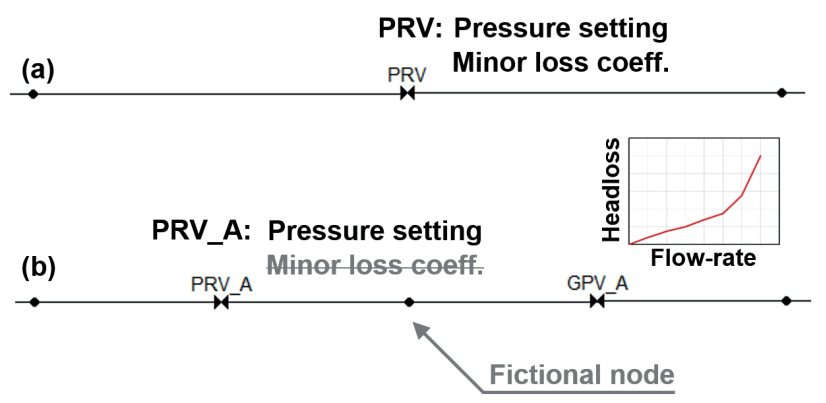

Fig. 3 PRV + GPV setup in a pure EPANET environment (a) original modeling approach (b) proposed setup 


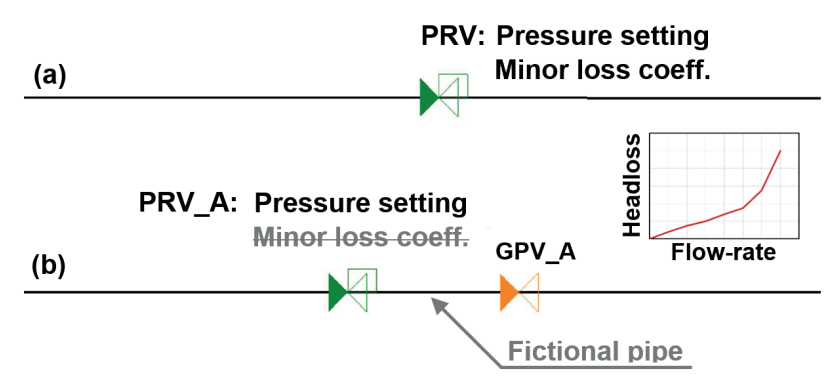

Fig. 4 PRV + GPV setup in WaterGEMS environment

(a) original modeling approach (b) proposed setup

Otherwise, their hydraulic behavior is the same, so the proposed calibration methodology is also valid in the WaterGEMS environment.

\section{Methodology}

\subsection{Calibration}

It is essential to accurately calibrate a hydraulic model to perform further analysis on it, like hydrant capacity analysis or chlorine residual modeling [10]. During the hydraulic model calibration the following assumptions were made:

- All the water demands are met because the minimum pressure was higher than the service pressure during the timeframe in question.

- Constant nodal demand weights are used based on the billed consumption.

- No pipe burst happened during the timeframe, which would alter operation based on the logs of the utility.

The following measurements were available for the pressure reducing valves:

- flow rate before the pressure reducing valve,

- pressure on the PRV's downstream pipe,

- pressure on the PRV's upstream pipe.

The timeframe of the available time-series started at 2017. 06. 01 and it was 10 days long. The resolution of the regular time-series was 30 minutes. The flow-rate and pressure values were time-weighted averages calculated from irregular samplings.

The time-series were divided into calibration and validation parts. The first 5 days out of 10 were used to calibrate the models. The last day was utilized to validate the calibrated model. These days were sequential; hence the day of the week was not stationary. The water demand patterns might change due to this aforementioned condition, but the head loss curves of the PRV's do not depend on the actual water demand.

It was assumed, that the demand weights used to distribute the zonal demand was constant during the simulating because the demand weights calculated from the annual or monthly billed consumption were considered stationary in time.

The number of free variables can be calculated from the number of inlet valves and the resolution of the GPV head loss-curves:

$D=n_{P R V}+\sum_{i=1}^{n_{P R V}} n_{\text {segment }, i}$,

where: $D$ : number of variables in the optimization problem, $n_{P R V}$ : number of $P R V^{\prime}$ 's to calibrate, nsegment, $i$ : the number of curve segments of the $i$ th $P R V$.

A custom performance function was written in python utilizing scikit-learn package [11] and EPANETTOOLS written by Pathirana [12]. EPANETTOOLS is based on EPANET version 2.0, which lacks functions to alter curves in-memory - version 2.2 is going to include these functions, which is not yet finalized and released. A custom INP altering method was implemented in python to overcome this limitation, although it requires the reloading of the .INP file before every iteration.

Four different unconstrained optimization methods were chosen to solve this multidimensional optimization problem. Constrained optimization methods were excluded from the research to have a more general result on optimization performance in case of large-scale problems. Multiple stopping criteria were set up in order to limit the optimization runtime, which is essential due to the usually limited available CPU capacity and time [13]. Function evaluation was limited to 1500 because different methods have a different number of function evaluation per iteration depending on the number of variables, thus using iteration limit would not have been suitable.

\subsubsection{Powell}

Powell's method [14, 15] doesn't require the loss function to be differentiable nor any derivatives are taken. It generates a sequence of points along each base vector. It is a robust, but generally inefficient due to the geometry of multivariable functions [16].

\subsubsection{Nelder-mead simplex method}

The Nelder-Mead is a simplex method [17, 18] also known as the downhill simplex method. A simplex consists of $\mathrm{N}+1$ vertices in an $\mathrm{N}$-dimensional search space. The hyperparameters can be adjusted in order to provide better performance in case of high-dimensional problems [19]. It is a widely-adopted optimization method, also present in MatLab. 
Multiple modifications were made on method, since it was originally published in 1965 including the Adaptive NelderMead and Restrced Nelder-Mead methods.

\subsubsection{BFGS}

BFGS is an implementation of the quasi-Newton method by Broyden, Fletcher, Goldfarb, and Shanno [20]. QuasiNewton-type methods might not converge in case of nonsmooth optimization, although BFGS was found to have acceptable performance even in these cases [19, 21]. BFGS is also widely-adopted in cases of unconstrained optimization and cases of nonlinear systems of equations.

\subsection{Operational performance assessment of a pressure managed zone}

Hydraulic modeling usually assumes ideal conditions, although these conditions are rarely met in real-life operation. There are several measures to analyze in order to get more detailed insight into the operation of a pressure reduced area:

- Pressure management index [22]

- Infrastructure Leakage Index (ILI)

- Inlet supply ratio - in case of multiple inlets

- Inlet valve performance

PMI is more useful during the design and commissioning phase of a pressure management task, but the latter two are more suitable to assess actual operational performance and detect anomalies during daily operation.

The maintenance requirements of mechanically operated pressure reducing valves vary by manufacturer and model. Although, there are recommendations about periodic maintenance, these do not include how often the valves should be vented due to the high uncertainty in gas ingress and movement in the system.

\subsubsection{Pressure management index}

The Pressure Management Index (PMI) is a performance indicator introduced by Trow [22]. It is usually used along with the Infrastructure Leakage Index (ILI). Both of these indicators' optimal value is 1.0 , which makes visualization and evaluation easier in a multi-objective optimization. Furthermore, their product is considered the Global Leakage Index (GLI) proposed by Renaud [23].

It gives a general overview on the performance of pressure management. Although, in gives no information about the remaining pressure reduction headroom in the system. If the difference between the minimum and maximum hydraulic grade demand is high in the pressure zone, the pressure reduction headroom is low.

\subsubsection{Inlet Supply Ratio}

The operation of the pressure reduced zones with multiple inlets can be characterized by the ratio of inlet flow-rates. The actual ratio depends on multiple factors, like:

- head loss curves of the valves,

- pressure setting of the valves,

- demand distribution in time,

- demand distribution along the network,

- total water demand of the area.

The ratio of flow-rates can be calculated by the following equation (Eq. (2)) for each time-step:

$$
\operatorname{Qrel}_{i, j}=\frac{Q_{i, j}}{\sum Q_{i, j}},
$$

where: Qrel: is the unitless supply ratio of valve $i$ in timestep $j Q_{i}$ : is the flow-rate of valve $i$ in timestep $j$.

As Fig. 5 indicates, the ratio of inlet flow-rates varies by the time during the day as water demand changes. The resolution of the graph was the same as the default time-step of the simulation: 30 minutes.

If the resolution is changed to 1 day (Fig. 6), the trend of the inlet supply ratio becomes smoother.

$$
\text { Qrel, } d_{i}=\frac{Q d_{i}}{\sum Q d_{i}},
$$

where: $Q r e l, d$ : is the unitless supply ratio of valve $i, Q d, i$ : is the average daily flow-rate of valve $i$.

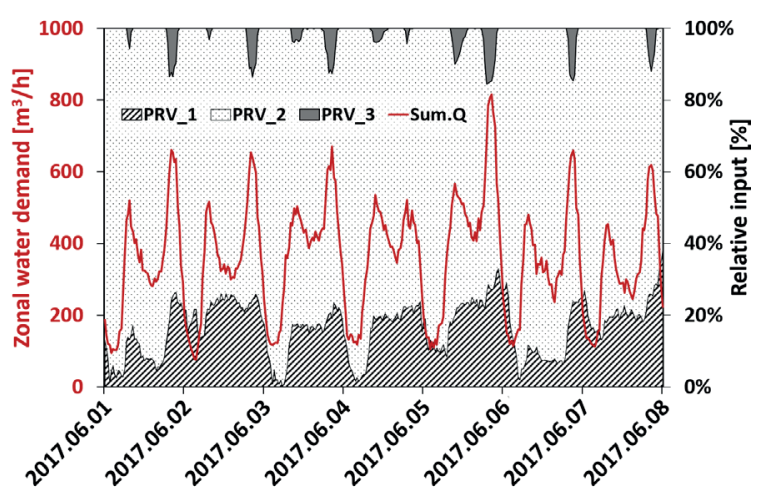

Fig. 5 Ratio of inlet flow-rates at a resolution of 30 mins

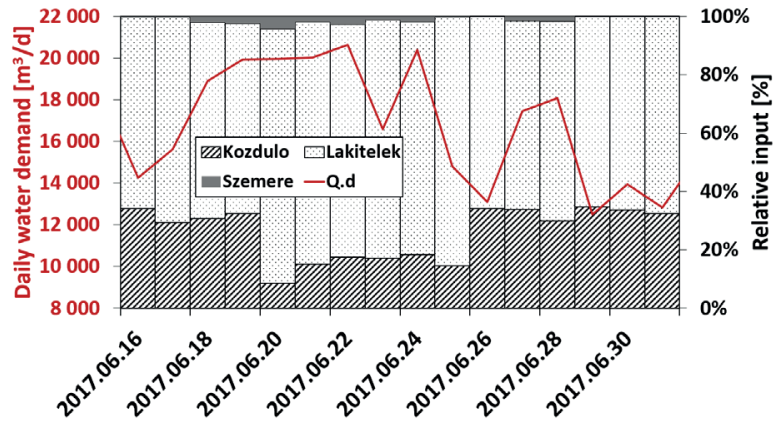

Fig. 6 Ratio of inlet flow-rates at a resolution of 1 day 
However, it is clear that the daily supply ratio is not proportional to the daily zonal water demand. The change in the daily supply ratio can indicate various events in the network. The movement of the demand's point of gravity due to bursts, leakage, planned reconstruction and segmentation or seasonal trends in water demand. It can also indicate changes in the performance of a valve due to air pocket accumulation, clogging or malfunction.

The change in valve performance can be determined by analyzing the head loss of the valve against the discharge flow-rate. If the additional head loss curve is stationary, no change in performance occurred. These events can be detected by time-series analysis. Any time-series anomaly detection method used on MNF (minimum night flow) series can be applied for this purpose.

It is essential to calibrate the model to fit the measured supply inlet ratios because mixing can affect water quality models directly [24] in case of different water sources are at the upstream side of the inlet PRV's.

\subsubsection{Valve performance evaluation}

The operation performance of pressure reducing valves can be described by the flow rate at which the downstream pressure head starts to drop below the pressure setting. This flow rate would be equal to the theoretical capacity of the valve in an ideal situation. The theoretical capacity is often characterized by the $K v$ (or $K c$ expressed in imperial units) value of the fully open valve.

$$
K v=Q \sqrt{\frac{G_{f}}{\Delta P}},
$$

where $K v$ : is the valve flow coefficient in $\mathrm{m}^{3} / \mathrm{h} / \mathrm{bar}^{0.5}, Q$ : is the flow rate in $\mathrm{m}^{3} / \mathrm{h}, G_{f}$ : is the liquid specific gravity (1.0 for water), $\Delta P$ : Differential pressure in bar.

It is possible to calculate the relative opening of the valve if the valve closure curve is available as some examples can be seen in Fig. 7 [25].

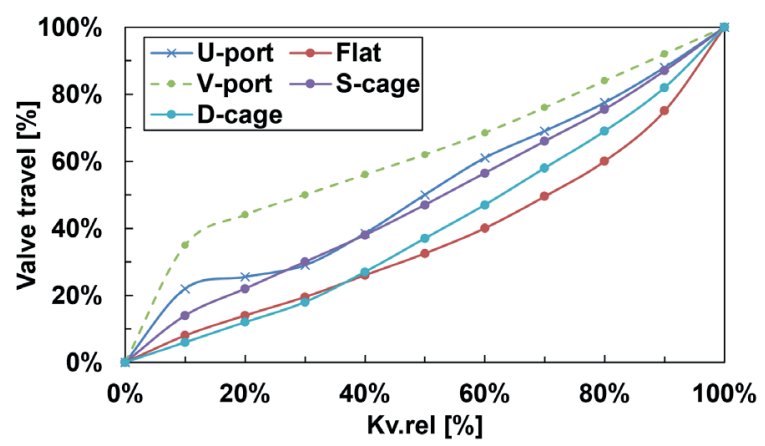

Fig. 7 Different closing characteristics of PRV's [25]
The pressure reducing valves have different closing characteristics depending on the shape of the orifice and the actuator. Real-life operational data at Budapest Waterworks showed that additional head loss (relative to the pressure setting) occurred even below $10 \%$ of the theoretical flow rate capacity - regardless of the manufacturer of the valve. The calculated relative valve opening percentage was higher due to the non-linear characteristic of the valves.

Table 1 summarizes the percentage of the theoretical capacity at which additional head loss was experienced compared to the pressure setting:

If the operating points of the valve are plotted against the theoretical head loss curve, the difference between the actual and the theoretical operation can be assessed. The evaluation of the operating points can be scalarized by fitting a linear function on them and taking the first-order coefficient, hence the first-order coefficient is a good indicator of operational performance. If the coefficient is near 0 , that means the valve performance is good on the observed flow-rate domain. If the coefficient's value is negative, that indicates additional pressure loss compared to the pressure setting.

It is important to note that other indicators can also represent the performance of a pressure reducing valve. The dimensionless cavitation index [26] or the number of valve closures and openings in one day.

\subsubsection{Effect of gas accumulation}

Water distribution networks are generally treated as a onephase, fully pressurized system. Although, air can ingress into the distribution network at specific events, like main burst, repairs or pressure deficiency. These events can be tracked, however, the movement of the gases cannot be tracked by the currently available tools.

These small air pockets usually exit the distribution network through the consumers' taps, hydrants or ventilation air valves at local high points. The PRV's characteristic

Table 1 Pressure reducing valve capacity based on their flow-rate coefficient

\begin{tabular}{lcccc}
\hline Curve_ID & Kv_full & Kv_calc & Kv_calc_rel & Valve_travel \\
\hline PRV_A & 519 & 36.9 & $7.1 \%$ & $28.1 \%$ \\
PRV_B & 336 & 9.7 & $2.9 \%$ & $14.2 \%$ \\
PRV_C & 519 & 60.0 & $11.6 \%$ & $37.0 \%$ \\
PRV_D & 336 & 37.8 & $11.3 \%$ & $36.5 \%$ \\
PRV_E & 336 & 9.1 & $2.7 \%$ & $13.5 \%$ \\
PRV_F & 55 & 10.2 & $18.6 \%$ & $44.0 \%$ \\
PRV_G & 55 & 2.1 & $3.8 \%$ & $17.8 \%$ \\
\hline
\end{tabular}


head loss curve can change, if these traveling air pockets reach the pressure reducing diaphragm valve they get trapped in the valve housing.

As Fig. 8 indicates, releasing accumulated gas pockets from the pressure reducing diaphragm valve is essential for a stable and reliable operation. Releasing trapped air is possible manually by opening a valve, or by automatic air-release valve.

If the change in the head loss curve is detected, a maintenance checkup can be assigned to the valve preventing the valve's subpar performance at peak demands. If air accumulation occurs during the low-demand season, it might not be detected until the additional head loss reach such a high level that can only occur during peak-demands. Preventive valve capacity check-ups should be performed by simulating high demands by opening hydrants in the PMA. Subpar valve capacity can be detected this way even before the consumer complaints would appear due to the pressure deficiency it causes.

\subsection{Commissioning of inlet valves}

It is essential to set the inlet pressure reducing valves to the same hydraulic grade in order to maintain a stable operation. If one of the valves stays closed until peak demands, when the head loss gets high enough in the networks. At certain settings, this means that a valve is closed for months. Eventually, in some cases, the flow in the particular pipe section can increase and considerably exceed the typical maxima, which can result in re-suspension of accumulated sediments and the occurrence of a discoloration incidents [27].

The following commissioning procedure describes a step by step approach to synchronize the inlet valves, which is inevitable to provide a stable and resilient operation (Fig. 9):

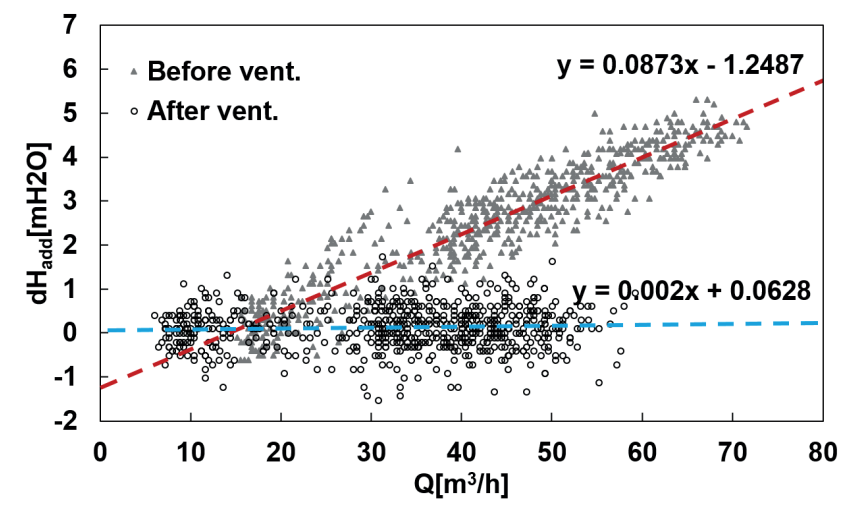

Fig. 8 Additional head loss of a PRV on the discharge side compared before and after air release. Trapped gases severely reduce the valve's capacity

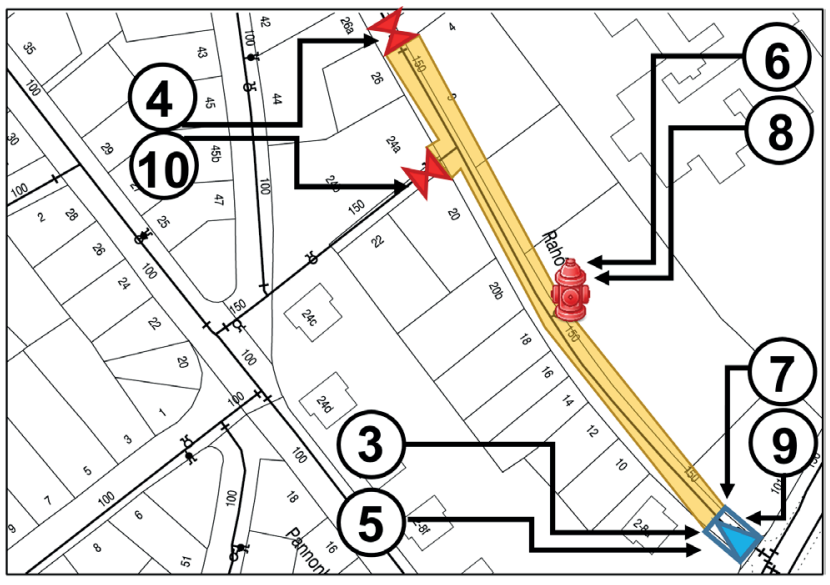

Fig. 9 Step-wise procedure to set the PRV's to the desired pressure setting in case of multiple inlet valves

- Step 1: Select the isolation valves and hydrants, which are necessary for the setting.

- Step 2: Calculating the target pressure settings by subtracting the elevation from the target hydraulic grade.

- Step 3: Check that air is released from all the inlet PRVs.

- Step 4: Segmentation on the downstream side of the PRV. The segment must include a hydrant or option to induce high flow-rates.

- Step 5: Set the PRV to the target pressure setting. Note, that the segmented area has low water demand.

- Step 6: Open the hydrant gradually, without exceeding the capacity of the valve. This induces higher discharge on the PRV.

- Step 7: Check if the valve can sustain the target pressure setting. Adjust the pilot if it is necessary.

- Step 8: Close the hydrant, hence the demand on the downstream side is reduced.

- Step 9: Check if the valve can sustain the target pressure setting.

- Step 10: Reopen the isolation valves, joining the separate sections of the network in the downstream side.

The aforementioned process can be repeated on every input PRV regardless of the number of them. No flow-rate measurement is required to perform the commissioning, although it is advised so, in order to monitor the operational performance of the valves. It has to be noted, that PMAs with a single inlet do not require to be segmented on downstream in order to accurately set the pressure setting to the target pressure setting.

The methodology was tested on three dual-inlet PMA's in real-life conditions for validation purposes (Fig. 10). 


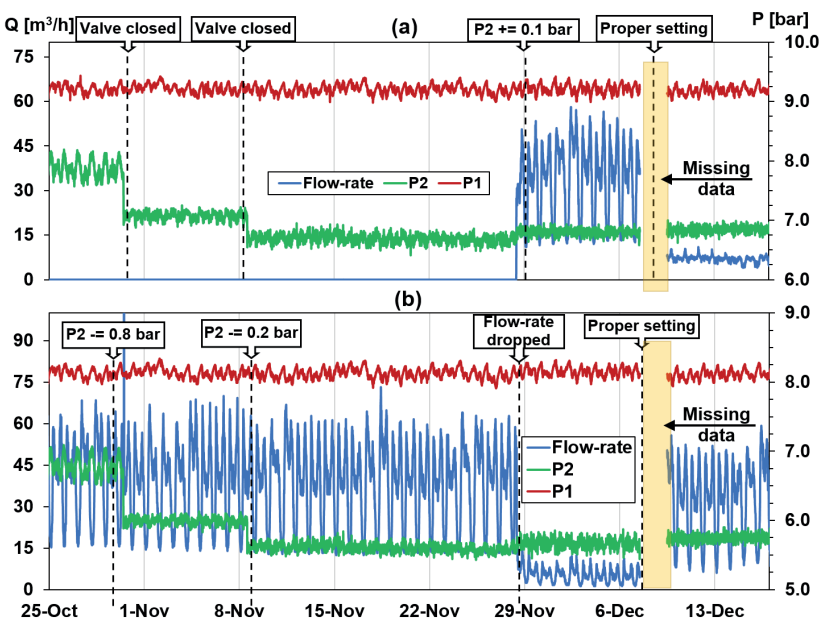

Fig. 10 Commissioning of a dual-inlet pressure reduced zone with the proposed methodology (a) PRV_A (b) PRV_B

\section{Results}

\subsection{Calibration results}

According to the calibration runs in a former study [8], using a simple quadratic head loss, there is a trade-off between the downstream pressure heads. The use of segmented quadratic head losses provided better results, but it is still far from the target calibration accuracy. It is possible to achieve a good fit on the measured values using the combined PRV+GPV approach. Unitless error measures, NRMSE and MAPE were used to evaluate the calibration performance because both pressure and flow-rate measurements were utilized during the calibration and validation. All of the optimization runs were terminated by the function evaluation criterion, which limited the maximum function evaluation in 1500. The stopping criterion is only checked at the end of each iteration, which is a limitation of the SciPy implementation. Some values might be higher than that if multiple function evaluations happen during a single iteration (Table 2).

The optimization runs duration lasted from 6 to $8 \mathrm{~min}$ utes that equals $\sim 3.2$ function evaluation per second. The temporary. INP write and rereads couldn't be omitted due to the limitations of the EPANET 2.0 toolkit. Although, the experimental EPANET 2.2 (OWA) version allows the user to skip the. INP format, which could increase the runtime performance.

The GPV head loss curves can be directly calculated from the measured discharge pressures by fitting a multipoint curve on it, although a multi-point curve seemed less robust due to the daily distribution of valve discharge. If all the necessary measurements are available, the utilization of meta-heuristic optimization methods can be omitted. If one of the downstream pressure measurements is missing, the unknown head loss curve can be calibrated using the aforementioned optimization methods.

\subsection{Operational performance results}

The operational performance results of multiple pressure managed areas are summarized in Table 3. No examined valve was in danger of damage caused by cavitation. Cavitation was prevented during the elaborate planning of the pressure managed areas and the localization of the inlet points.

\section{Discussion}

\subsection{Calibration}

The validation errors were higher than the errors measured on the calibration dataset comparing the results in Table 2 and Table 3.

The reason behind it is that the same nodal demand weights were used during the simulations, although the spatial distribution of water demand might change during the week.

Both the adaptive Nelder-Mead and the Powell direct search methods improved the fit on the measured datasets. Although, the Powell method seems less susceptible to find a local minimum than the adaptive Nelder-Mead (Fig. 11). The use of the BFGS and TNC methods did not improve significantly the fitness of the model compared to the reference.

\subsection{Valve performance evaluation}

Pressure managed areas with multiple inlets require special care during design, commissioning, and operation. Commissioning is possible with temporary pressure and

Table 2 PRV Calibration results

\begin{tabular}{lccccc}
\hline & $\begin{array}{c}\text { Nelder- } \\
\text { Mead }\end{array}$ & Powell & BFGS & TNC & Reference \\
\hline NRMSE & 0.1680 & 0.1462 & 0.2216 & 0.2091 & 0.2216 \\
MAPE & 0.0964 & 0.0895 & 0.1255 & 0.1158 & 0.1255 \\
GOF & 0.8799 & 0.8791 & 0.8376 & 0.8376 & 0.8376 \\
Iterations & 1065 & 5 & 2 & 1 & - \\
$\begin{array}{l}\text { Function } \\
\text { evaluation }\end{array}$ & 1501 & 2567 & 1438 & 342 & - \\
$\begin{array}{l}\text { Duration [sec] } \\
\text { Nution }\end{array}$ & 613 & 1181 & 639 & 145 & - \\
\hline
\end{tabular}

Table 3 PRV Validation results

\begin{tabular}{lccccc}
\hline & Nelder-Mead & Powell & BFGS & TNC & Reference \\
\hline NRMSE & 0.2427 & 0.2602 & 0.3007 & 0.3007 & 0.3007 \\
MAPE & 0.1382 & 0.1453 & 0.1520 & 0.1520 & 0.1520 \\
GOF & 0.7871 & 0.7832 & 0.7575 & 0.7575 & 0.7575 \\
\hline
\end{tabular}




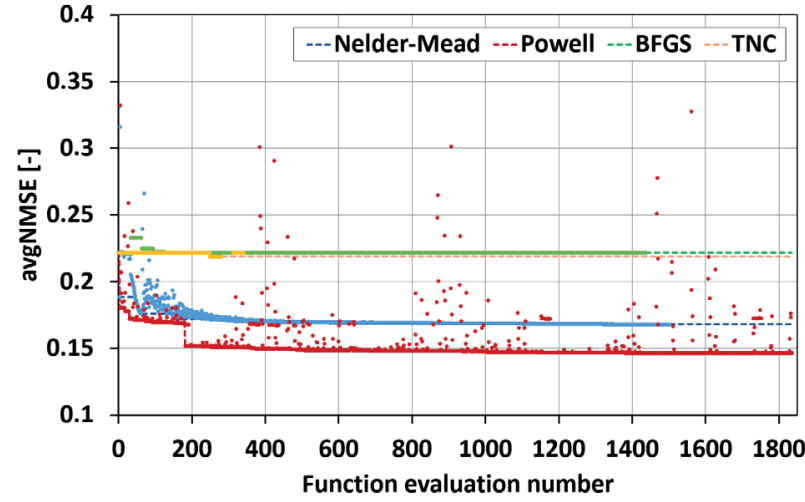

Fig. 11 Function Loss value propagation during optimization

flow-rate measurements, but the support of operation and maintenance can only be achieved by installed meters integrated into the SCADA system. The lack of instrumentation makes harder to set the valves to their target pressure settings, which was determined during the design phase. If additional pressure loss occurs during normal operation and water demand, the synchronization of the valves is exceptionally challenging, especially if the valves lack pressure or flow measurements.

If the inlet valve lacks pressure measurement upstream, it prohibits the calculation of $\Delta P$, hence there the risk of cavitation cannot be assessed.

Table 4 summarizes, which tasks can be achieved at certain instrumentation levels:

The head loss-curve of the PRVs is an important indicator of valve operational performance and actual capacity. Sub-par performance can be detected by evaluating these curves, hence it is possible to correct it by maintenance, even before customer complaints would directly

Table 4 Measurements required for detecting specific phenomenon related to pressure reducing valves

\begin{tabular}{lccccc}
\hline $\begin{array}{l}\text { Available } \\
\text { measurements }\end{array}$ & Altering & Cavitation & Perf. & Offset & Capacity \\
\hline $\mathrm{Q}$ & $\mathrm{X}$ & & & & \\
$\mathrm{P} 1+\mathrm{Q}$ & $\mathrm{X}$ & & & & \\
$\mathrm{P} 1+\mathrm{P} 2$ & & $\mathrm{X}$ & & & \\
$\mathrm{Q}+\mathrm{P} 2$ & $\mathrm{X}$ & & $\mathrm{X}$ & $\mathrm{X}$ & \\
$\mathrm{Q}+\mathrm{P} 1+\mathrm{P} 2$ & $\mathrm{X}$ & $\mathrm{X}$ & $\mathrm{X}$ & $\mathrm{X}$ & $\mathrm{X}$ \\
$\mathrm{Q}+\mathrm{P} 1+\mathrm{P} 2+\mathrm{h}$ & $\mathrm{X}$ & $\mathrm{X}$ & $\mathrm{X}$ & $\mathrm{X}$ & $\mathrm{X}$ \\
\hline
\end{tabular}

\section{References}

[1] Farley, M. "Leakage management and control: a best practice training manual", World Health Organization, Geneva, Switzerland, 2001. [online] Available at: https://apps.who.int/iris/handle/10665/66893 [Accessed: 28 March 2020] indicate the need for maintenance. The curve evaluation was performed manually, but automated anomaly detection performed on these measured head loss curves holds great potential.

It has to be noted that the hydraulic simulations were conducted assuming steady-state conditions. This boundary condition prevents us from implementing hysteresis curves, which affects the operating point of the valve [28].

\section{Conclusions}

On-site operational observations show that a single PRV object cannot describe the actual behavior of pressure reducing valves if there are more than one inlets of the pressure managed area. This trade-off makes the proper commissioning of these PMA's harder, especially if the level of instrumentation is low. The proposed methodology to commission a multi-inlet PMA is suitable to accurately set the discharge pressure setting for each inlet valve separately, hence a more stable operation can be ensured. The method described does not require to have on-line flow rate measurements at the inlet points, since the target hydraulic grades can be synchronized by the manual downstream pressure metering.

Hydraulic modeling and calibration require a PRV + GPV setup in order to accurately represent the real behavior of these systems in an EPANET-forked environment. Metaheuristic non-constrained optimization methods are suitable to calibrate the multi-point head loss curves and actual pressure setting of the valves. The adaptive NelderMead and the Powell algorithm provided better results than the others. However, steady-state hydraulic modeling is not suitable to take into account the hysteresis and closing or opening characteristics of these valves, which is the reason behind the scattered discharge pressure values during apparently the same conditions.

\section{Acknowledgment}

The authors gratefully acknowledge the contributions and support of several individuals at Budapest Waterworks who assisted with on-site measurements, data processing: Evelin Madzin, Viktor Horváth.

[2] Kanakoudis, V., Gonelas, K. "Applying Pressure Management to Reduce Water Losses in Two Greek Cities' WDSs: Expectations, Problems, Results and Revisions", Procedia Engineering, 89, pp. 318-325, 2014. https://doi.org/10.1016/j.proeng.2014.11.194 
[3] Fallis, P., Hübschen, K., Oertlé, E., Ziegler, D., Klingel, P., Knobloch, A., Baader, J., Trujillo, R., Laures, C. "Guidelines for water loss reduction - A focus on pressure management", Deutsche Gesellschaft für Internationale Zusammenarbeit (GIZ) $\mathrm{GmbH}$, Eschborn, Germany, 2011.

[4] Adedeji, K. B., Hamam, Y., Abe, B. T., Abu-Mahfouz, A. M. "Pressure Management Strategies for Water Loss Reduction in Large-Scale Water Piping Networks: A Review", In: Gourbesville, P., Cunge, J., Caignaert, G. (eds.) Advances in Hydroinformatics SimHydro 2017—Choosing the Right Model in Applied Hydraulics, Springer, Singapore, 2018, pp. 465-480.

https://doi.org/10.1007/978-981-10-7218-5_33

[5] Giustolisi, O., Ugarelli, R. M., Berardi, L., Laucelli, D. B., Simone, A. "Strategies for the electric regulation of pressure control valves", Journal of Hydroinformatics, 19(5), pp. 621-639, 2017. https://doi.org/10.2166/hydro.2017.101

[6] Xu, Q., Chen, Q., Ma, J., Blanckaert, K., Wan, Z. "Water Saving and Energy Reduction through Pressure Management in Urban Water Distribution Networks", Water Resources Management 28, pp. 3715-3726, 2014.

https://doi.org/10.1007/s11269-014-0704-1

[7] Samir, N., Kansoh, R., Elbarki, W., Fleifle, A. "Pressure control for minimizing leakage in water distribution systems", Alexandria Engineering Journal 56(4), pp. 601-612, 2017.

https://doi.org/10.1016/j.aej.2017.07.008

[8] Bibok, A., Fülöp, R. "Hydraulic Model Calibration of Pressure Reduced Zones with Multiple Input Valves", In: La Loggia, G., Freni, G., Puleo, V., De Marchis, M. (eds.) HIC 2018. 13th International Conference on Hydroinformatics, 3, 2018, pp. 274-281. https://doi.org/10.29007/8vqn

[9] Bentley Systems "WaterGEMS User's Guide", Bentley Systems, Inc., Exton, PA, USA, 2019.

[10] Al Heboos, S., Licskó, I. "Application and Comparison of Two Chlorine Decay Models for Predicting Bulk Chlorine Residuals", Periodica Polytechnica Civil Engineering, 61(1), pp. 7-13. 2017. https://doi.org/10.3311/PPci.9273

[11] Pedgerosa, F., Varoquaux, G., Gramfort, A., Michel, V., Thirion, B., Grisel, O., Blondel, M., Prettenhofer, P., Weiss, R., Dubourg, V., Vanderplas, J. T., Passos, A., Cournapeau, D., Brucher, M., Perrot, M., Duchesnay, É. "Scikit-learn: Machine Learning in Python", The Journal of Machine Learning Research, 12, pp. 2825-2830, 2011. https://dl.acm.org/doi/10.5555/1953048.2078195

[12] Pathinara, A. "EPANETTOOLS - EPANET calling API for python", [online] Available at: https://github.com/asselapathirana/epanettools [Accessed: 20 Oct 2019]

[13] Bene, J. G., Selek, I. "Water network operational optimization: Utilizing symmetries in combinatorial problems by dynamic programming", Periodica Polytechnica Civil Engineering, 56(1), pp. 51-61, 2012.

https://doi.org/10.3311/pp.ci.2012-1.06

[14] Powell, M. J. D. "An efficient method for finding the minimum of a function of several variables without calculating derivatives", The Computer Journal, 7(2), pp. 155-162, 1964.

https://doi.org/10.1093/comjn1/7.2.155
[15] Press, W. H., Teukolsky, S. A., Vetterling, W. T., Flannery, B. P. "Numerical Recipes - The Art of Scientific Computing", 3rd ed., Cambridge University Press, New York, NY, USA, 2007.

[16] Mathews, J. H., Fink, K. K. "Numerical Methods Using Matlab", 4th ed., Pearson, Upper Saddle River, NJ, USA, 2004.

[17] Nelder, J. A., Mead, R. "A Simplex Method for Function Minimization", The Computer Journal, 7(4) pp. 308-313, 1965. https://doi.org/10.1093/comjn1/7.4.308

[18] Wright, M. "Direct search methods: Once scorned, now respectable", In: Numerical Analysis: Proceedings of the 1995 Dundee Biennial Conference in Numerical Analysis, Nethergate, Dundee, Scotland, UK, 1996, pp. 191-208.

[19] Gao, F., Han, L. "Implementing the Nelder-Mead simplex algorithm with adaptive parameters", Computational Optimization and Applications, 51, pp. 259-277, 2012. https://doi.org/10.1007/s10589-010-9329-3

[20] Nocedal, J., Wright, S. J. "Numerical Optimization", 2nd ed., Springer, New York, NY, USA, 2006. [online] Available at: https://www.csie.ntu.edu.tw/ r97002/temp/num_optimization.pdf [Accessed: 28 March 2020]

[21] Bonnans, J.-F., Gilbert, J. C., Lemaréchal, C., Sagastizábal, C. A. "Newtonian Methods", In: Numerical Optimization: Theoretical and Practical Aspects, 2nd ed., Springer, Berlin, Germany, 2006, pp. $51-66$. https://doi.org/10.1007/978-3-540-35447-5

[22] Trow, S. W. "Development of a Pressure Management Index", presented at IWA Water Loss 2009 Conference, Cape Town, South Africa, April, 26-30, 2009.

[23] Renaud, E. "Towards a global performance indicator for losses from water supply systems", presented at IWA Water Loss 2010 Conference, Sao Paulo, Brazil, June, 6-9, 2010.

[24] Szuster-Janiaczyk, A., Brodziak, R., Bylka, J. "The use of mathematical modelling to analyse the process of mixing waters from different sources in water supply system", presented at E3S Web of Conferences, 2nd International Conference on Science and Technology Current Issues in Water Distribution and Treatment (CIWT 2017), 59, Article Number: 00025, 2018. https://doi.org/10.1051/e3sconf/20185900025

[25] Bermad Water Technologies "720 Series Engineering Data", Water Control Solutions, Houston, TX, USA. [online] Available at: https:// www.bermad.com/wp-content/uploads/WW_700-SIGMA_engineering_English_v22.pdf [Accessed: 16 Jan 2020]

[26] ISA "ISA-RP75.23-1995Considerations for Evaluating Control Valve Cavitation", International Society of Automation, Durham, NC, USA, 1995.

[27] Ručka, J., Kovar, J. "Prevention of Discoloration Events in Water Distribution Systems", Procedia Engineering, 70, pp. 1441-1449, 2014.

https://doi.org/10.1016/j.proeng.2014.02.159

[28] Talamini, Jr., M. V., de Araujo, A. C. S., Camargo, A. P., Saretta, E., Frizzone, J. A. "Operational Characterization of Pressure Regulating Valves", The Scientific World Journal, 2018, Article ID: 1213638, 2018.

https://doi.org/10.1155/2018/1213638 